\title{
Chronobiology in Asia
}

\author{
Ken-ichi Honma ${ }^{1}$
}

Published online: 13 June 2016

(C) Japanese Society of Sleep Research 2016

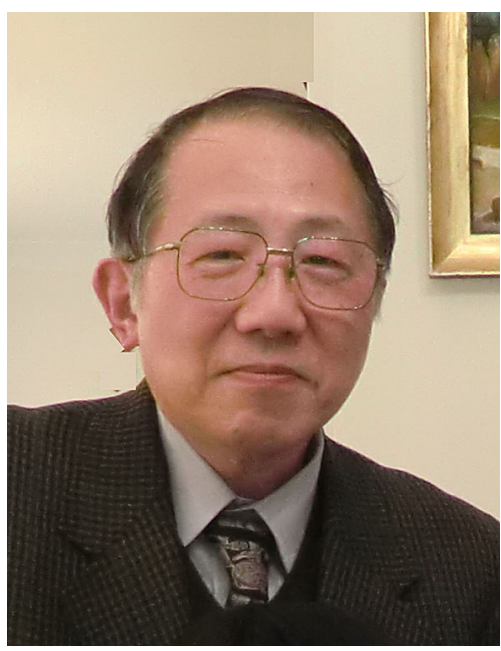

Chronobiology and sleep research are the two sides of a coin. Sleep and biological rhythms (SBR) is emphasizing this aspect, since both sciences have been relatively independent. This tradition is changing rapidly in Asia. As mentioned in the previous preface of SBR, chronobiology, a study on circadian rhythms and related phenomenon, has been growing in Asia. Last September, the first meeting of Asian Forum on Chronobiology (AFC) was held in Sapporo, Japan, and more than 50 scientists in this field were invited from China, Korea, India, Turkey and Japan. The meeting was so successful that the participants agreed with the continuation of such meeting to exchange information and ideas for future collaboration. As a result, the second

Ken-ichi Honma

kenhonma@med.hokudai.ac.jp

1 Hokkaido University Graduate School of Medicine, Sapporo, Japan
AFC was decided to be held in Antalya Turkey in 2016 and the third one in Soochow, China in 2017. In China, immediately after the meeting in Sapporo, more than 100 researchers on biological rhythms got together in Shi-JiaZhuang, China, and established a new society for biological rhythm research a branch society of Chinese Society for Cell Biology. On the same year, an Asian Cold Spring Harbor Symposium was held in Soochow for the first time in special reference to chronobiology. The meeting was organized by Dr. Han Wang in Soochow. In 2016, chronobiology is explored in China. In March 2016, many important studies were presented in special lectures, symposiums and posters at the 9th annual meeting of Chinese Society of Sleep Research in Shanghai, organized by Dr. Zhi-Li Huang. In August 2016, the International Summer School on Chronobiology will be held in Beijing for one week with an initiative of Dr. Ying Xu in Soochow, in hoping a success similar to, or more than that in Sapporo 2 years ago, organized by Dr. Sato Honma and myself. In Sapporo, more than 40 students and young scientists participated in the summer school not only from Asian countries but also from USA, Germany and Mexico. In October 2016, the biannual meeting of ISC (International Society for Chronobiology) will be held in Suzhou, organized by Dr. Han Wang. Most striking features of Chinese chronobiology are the molecular aspects of circadian rhythms and the participation of many students and young scientists in this field. In 2017, the 3rd Asian Forum on Chronobiology, and in 2018, the 5th World Congress of Chronobiology will be held in Suzhou. Now, Suzhou is the center of chronobiology in China.

In the present year (2016), the ASRA meeting will be held in Antalya, Turkey from October 1 to October 4 in association with the annual meeting of TSSM and 2nd AFC. On this occasion, we will select a new president and 
council members of ASRS. I hope a new cabinet to facilitate communication between sleep and biological rhythm researchers. Such attempts have been carried out in Japan for several times and will be repeated in future. In 2018, the ASRS meeting will be held in Sapporo in association with the 4th AFC and the annual meeting of Japanese Society of Sleep Research.

Physiologically, sleep and wakefulness are located in the downstream of circadian clock but the timing of sleep feedbacks onto the circadian clock, by gating the photic inputs into the circadian clock. Recently, physical exercise was reported to affect the circadian clock probably through changing the sensitivity to photic signals. The quality of wakefulness may influence the photic entrainment of circadian clock. Furthermore, the sleep/wake cycle could be an output of the extra-SCN circadian oscillations in the brain. Clinically, circadian rhythm disorders and sleep disturbance are closely related. Therefore, the target of integrated research on sleep and biological rhythm is abundant. Projects as well as researchers in this direction are growing in Asia. 